\title{
Accuracy of Sentinel Lymph Node Biopsy after Neoadjuvant Chemotherapy in Breast Cancer Patients; Single Center Experience
}

\author{
Waleed Elnahas' ${ }^{1}$, Omar Hamdy ${ }^{1}$, Khaled Abdel Wahab ${ }^{1 *}$, Sameh Roshdy ${ }^{1}$, Sara Raafat ${ }^{2}$, \\ Mohamed Hafez ${ }^{1}$, Omar Farouk ${ }^{1}$, Mohamed Elmetwally ${ }^{1}$ \\ ${ }^{1}$ Surgical Oncology Unit, Mansoura Oncology Centre, Mansoura University, Mansoura, Egypt \\ ${ }^{2}$ Pathology Department, Faculty of Medicine, Mansoura University, Mansoura, Egypt \\ Email: *khaled14eg@hotmail.com
}

How to cite this paper: Elnahas, W., Hamdy, O., Wahab, K.A., Roshdy, S., Raafat, S., Hafez, M., Farouk, O. and Elmetwally, M. (2018) Accuracy of Sentinel Lymph Node Biopsy after Neoadjuvant Chemotherapy in Breast Cancer Patients; Single Center Experience. Surgical Science, 9, 52-61.

https://doi.org/10.4236/ss.2018.91006

Received: December 8, 2017

Accepted: January 28, 2018

Published: January 31, 2018

Copyright $\odot 2018$ by authors and Scientific Research Publishing Inc. This work is licensed under the Creative Commons Attribution International License (CC BY 4.0).

http://creativecommons.org/licenses/by/4.0/

\section{(c) (i) Open Access}

\begin{abstract}
Purpose: Most important factor affecting prognosis of breast cancer is axillary nodal involvement. Several studies evaluated the accuracy of Sentinel lymph node biopsy in breast cancer patients post neoadjuvant chemotherapy. In this study, we will examine accuracy and feasibility of using Sentinel lymph node biopsy in predicting axillary lymph node status in breast cancer patients after neoadjuvant chemotherapy. Methods: 45 female patients with resectable, nonmetastatic breast carcinoma cases who received neoadjuvant chemotherapy were enrolled in this study according to the routine Mansoura Oncology Center-guidelines of management of breast cancer. Methylene blue dye used for detection of Sentinel lymph node. Results: Successful Sentinel lymph node detection was $82.2 \%$. Skin involvement (T4 disease) were linked to a low identification $(P=0.005)$. False negative rate equals $11 / 27=(40.7 \%)$.With advancement of the stage of the tumor, the incidence of false negative results increases significantly $(p=0.012)$ with $95 \%$ confidence interval; $1.2-5.4$. Conclusion: Sentinel lymph node should be adopted to be the standard method for axillary staging with T1-3 tumors after receiving neoadjuvant chemotherapy, in T4 patients, it is associated with low detection rate $\&$ high false negative rate making it doubtful technique for axillary staging.
\end{abstract}

\section{Keywords}

Breast Cancer, Sentinel Lymph Node, Neo Adjuvant Chemotherapy, Blue Dye

\section{Introduction}

The prognosis of breast cancer patients depends mainly on many factors in- 
cluding tumor characteristics especially its size, histological grade, hormonal receptor status \& patients age at the time of diagnosis as well as axillary nodal status. However, the most important factor among all of these is axillary nodal involvement, which carries a major impact on 5-year survival rate [1].

For axillary nodal involvement, axillary lymph node dissection (ALND) was considered the optimal care. But, it was also linked with several problems including lymphedema, numbness, pain, impaired shoulder girdle movement and overall affection of quality of life. These complications were a driving force towards a less invasive technique for axillary staging [2].

For years now, Sentinel lymph node (SLN) biopsy in early breast cancer is a routine practice as it provides an accurate \& less morbid method for staging preventing the undesirable problems of routine axillary lymph node clearance [3].

Nowadays, neoadjuvant chemotherapy gained popularity and became the first option in dealing with locally advanced breast cancer as well as its progressive usage with $\mathrm{T} 2$ breast cancer. Although there was no increase in disease-free survival or overall survival, neoadjuvant chemotherapy has been proven to carry many advantages such as downstaging of inoperable disease \& facilitating respectability and conservative breast surgery, in vivo testing of systemic therapy modalities [4] [5].

Accuracy and feasibility of using SNL biopsy after administration of neoadjuvant chemotherapy is debatable issue; some authors postulated that chemotherapy may change the lymphatic pathway, which can affect on the identification rate [6].

Several studies have assessed the accuracy and feasibility of this technique, but diagnostic accuracy has been varied [7].

In the present study, we will evaluate the feasibility and reliability of SLNB in predicting axillary lymph node status in breast cancer patients after neoadjuvant chemotherapy.

\section{Methods}

This is a prospective clinical study done during the period from September 2014 to May 2016 where 45 female patients with resectable, nonmetastatic breast carcinoma cases who received neoadjuvant chemotherapy and appeared to have negative axillae clinically and by radiological assessment were enrolled according to the routine Oncology Center-Mansoura University's guidelines of management of breast cancer. Approval from the ethical committee in Faculty of medicine, Mansoura University was obtained before starting the trial.

Patients with the following criteria were excluded from the study:

- Unfit patients for general anaesthesia.

- Previous axillary surgery.

- History of breast surgery or incisional biopsy compromising the breast lymphatic drainage. 
- Known hypersensitivity to the dye.

- Patients who received neoadjuvant hormonal therapy.

- Patients with clinicaly positive or suspicious axilla after neaodjuvant therapy All Patients were assessed carefully on clinical and radiological basis before administration of neoadjuvant chemotherapy.

All patients received doxorubicin and/or Taxan-based neoadjuvant chemotherapy. Management strategies for those patients were reviewed at the weekly multi-disciplinary breast team in Mansoura oncology center.

Patients who satisfied the inclusion criteria were subjected to breast surgery after discussing with the patient and explaining all the available procedures (modified radical mastectomy, conservative breast surgery or immediate reconstruction), sentinel lymph node biopsy and classic axillary lymph node dissection.

Intraoperative lymphatic mapping was performed after periareolar injection of 2 to $10 \mathrm{~mL}$ of sterile methylene blue dye, and then breast massage for $15 \mathrm{mi}$ nutes. After the administration of anesthesia, the planned incision was marked on the skin of the affected breast.

An incision planned just below the axillary hairline to be used as the same incision for axillary dissection in patients planned for breast conserving surgery or to be extended to perform the mastectomy incision in other cases that were planned to perform a more radical surgery.

The blue stained lymphatic pathways were identified (Figure 1) and followed up to the blue SNL which was identified (Figure 2 and Figure 3 ) by its color and sent for frozen section.

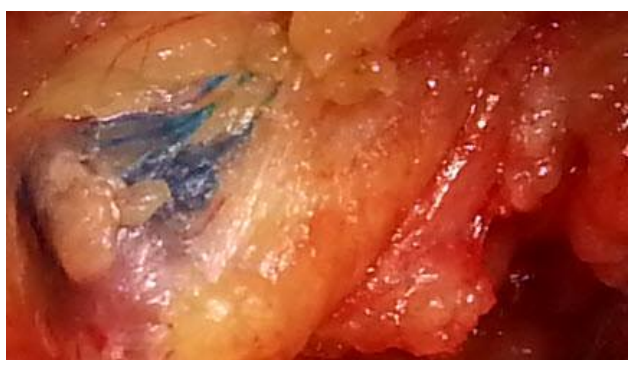

Figure 1. Sentinel LN with blue colored lymphatics passing towards it.

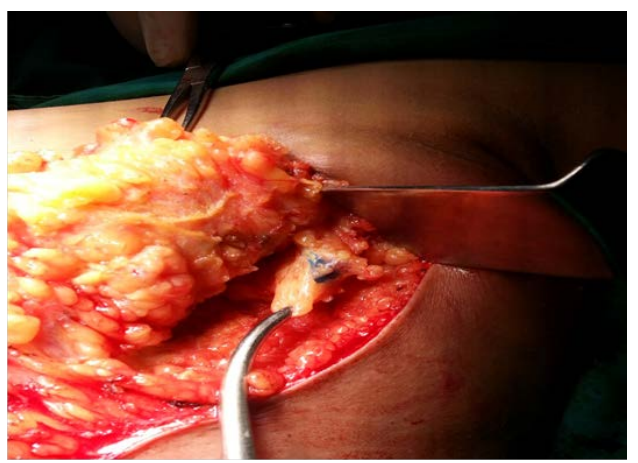

Figure 2. Sentinel LN identification. 


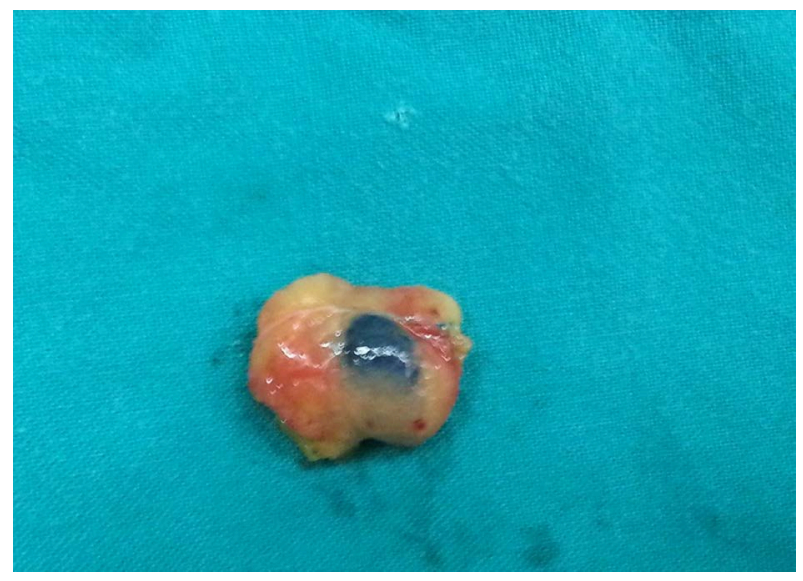

Figure 3. Specimen including single sentinel LN for HPE.

\section{Results}

The median patient age at the time of diagnosis was 50.2 years (20 - 71).

As regard the primary tumor size before and after receiving neoadjuvant chemotherapy; out of the 45 patients, 23 were T4 before starting chemotherapy, 8 were T3, 13 were T2 and only one was T1 (reported to be metastatic from the time of diagnosis, proved later on to be non-metastatic) with percentage of cases about $51.1 \%, 17.7 \%, 28.8 \%$, and $2.2 \%$ respectively. After receiving neoadjuvant therapy 4 patients achieved complete clinical response, 12 became T1, number of T2 tumors became 18, T3 and T4 tumors was only one and 10 consequently (Table 1).

As regard the state of lymph node pre and post receiving neoadjuvant chemotherapy; 18 patients were node negative, 24 were N1, 2 were $\mathrm{N} 2$ and one was N3a. All the patients were node negative after neoadjuvant therapy.

As regard the tumor pathological response to chemotherapy, 40 patients had residual malignant disease after preoperative chemotherapy, and, in five patients, there was a complete pathological response to chemotherapy. Those forty patients were listed as follow according to their histopathological type: 33 with IDC, 3 patients with invasive lobular carcinoma, 2 patients with DCIS, 1 patient with colloid carcinoma and another one with metaplastic carcinoma.

All 45 patients underwent attempted lymphatic mapping with methylene blue dye and SLN biopsy. Overall, there were 37 successful SNL detection (82.2\%) and eight unsuccessful detection (17.8\%). Univariate analysis of clinicopathologic features revealed that persistent skin involvement (T4 disease) after neoadjuvant therapy was linked significantly with a decreased probability to identify SLN $(P=0.005)$. if T1-3 \& T4 tumors (after NAC) identification rate was calculated independently, the result will differ as follows; T1-3 tumors (after NAC); SLN was identified in 34 out of 35 patients giving percentage of $97.1 \%$ while in T4 tumors (after NAC); SLN was identified only in 3 out of 10 patients giving percentage of $30 \%$. Patient age, tumor site and initial lymph node tumor burden were not correlated with successful lymphatic identification. 
Table 1. Illustrates the tumor and lymph node staging before and after neo adjuvant chemotherapy characteristics.

\begin{tabular}{ccc}
\hline T stage & Before chemotherapy (no) & After chemotherapy (no) \\
\hline T0 (cCR) & 0 & 4 \\
T1 & 1 & 18 \\
T2 & 13 & 1 \\
T3 & 8 & 10 \\
T4 & 23 & After chemotherapy (no) \\
N stage & Before chemotherapy (no) & 45 \\
N0 & 18 & 0 \\
N1 & 24 & 0 \\
N2 & 2 & 0 \\
N3 & 1 & \\
\hline
\end{tabular}

Among the 37 cases where SLN could be detected and sent for histopathological examination, the result in 27 patients was negative for tumor deposits while was positive in 10 patients only (one of them showed micrometastasis) and it was the only positive node in one patient.

On the other hand, as regard the final pathology of axillary lymph node dissection (ALND), 17 patients showed negative axillae, while 20 patients showed positive tumor deposits in the dissected axillary LNs. One of them was the only positive node.

This means that the total false negative rate equals $11 / 21=(52 \%)$.

Calculating false negative results according to the stage revealed that with advancement of the stage of the tumor, the incidence of false negative results increases significantly $(P=0.012)$ with $95 \%$ confidence interval; $1.2-5.4$; When false negative rate was calculated separately for patients who were T4 before NAC, the results were as follow: T4 group: fNR equals $9 / 13=69 \%$ while in T1-3 group: fNR equals $2 / 7=28 \%$.

The accuracy of frozen section in examination of sentinel LNs was $100 \%$. All the frozen section results were exactly as the paraffin.

No allergic reactions, short or long-term complications were recorded for the maneuver.

\section{Discussion}

Sentinel lymph node (SLN) biopsy in early breast cancer is referred to nowadays as the standard of care as it provides an accurate \& less morbid method for staging avoiding the undesirable complications of routine axillary clearance [3].

SLN identification can be performed with equal success rates using methylene blue and patent blue dyes. Methylene blue can be used as a cost-effective technique in economically less developed countries [8].

Methylene blue dye is a cheap, efficient alternative for patent blue dye with 
less allergic reactions in performing sentinel LN biopsy.

The technique was first described for axillary staging in breast cancer using blue dye in 1994 by Giuliano et al. with identification rate in $66 \%$ of cases \& accuracy rate of $96 \%$ [9].

Usage of Gamma probe to identify SLN was performed in 1993 by Krag et al. $82 \%$ identification rate [10].

It has also been proved that the combination between two methods is much more sensitive, specific \& accurate in prediction of the axillary nodal status [11].

As regard the method used for lymphatic mapping in our study, it was methylene blue due to non-availability of either radioactive material or patent blue dye.

Although the combined technique offers more rapid identification of SLN, the methylene blue dye technique alone can be used efficiently in centers which do not own nuclear medicine facilities [12].

Neoadjuvant chemotherapy was considered as a contraindication to SLNB. Schwartz et al, in 2003, suggested that neoadjuvant chemotherapy should not be an absolute contraindication to SLNB. Their study conducted on 21 patients showed an identification percent of $95 \%$ and a false negative result in only one patient [13].

The first published meta-analysis was that of Xing et al. They included articles discussing the issue of the accuracy of sentinel lymph node biopsy in cases that have received neo adjuvant chemotherapy. Articles that fit their inclusion criteria were 21 comprising about 1273 patients. The identification rates reported ranged from 72 to $100 \%$, with reported sensitivity of about $67 \%$ to $100 \%$. They stated at the end of their study that we can use SNLB in patients that had received neoadjuvant chemotherapy with good reliability [14].

Also, Kelly and his team collected results of about 24 trials with a total number of cases of about 1799 subjects. They found an identification rate of about $89.6 \%$ with the false-negative rate was about $8.4 \%$. They had a conclusion that such a technique after neoadjuvant chemotherapy showed high accuracy and feasibility for axillary staging [15].

This was nearly the same conclusion that was stated by Van Deurzen et al. where the SLN identification rate was $90.9 \%$ with false-negative rate was about $10.5 \%$. They just added that there is still inadequate evidence to recommend it as the standard procedure [16].

A Prospective German, multi-institutional SENTINA-Trial performed on cases that were converted from positive to negative nodes post neo adjuvant chemo therapy declared that detection rate for the SLNB was significantly lower when these cases were compared to others who underwent SLNB before any other treatment. They clearly stated in their conclusion that administration of systemic therapy significantly affects the accuracy of SLNB [17].

Issues that may affect detection of sentinel node biopsy after neoadjuvant chemotherapy may be: a proposed change in the lymphatic channels post systemic therapy. Recent study showed that although the location of sentinel nodes 
was not affected by NAC, nearly one third of the sentinel lymphatic pathways were modified [18]. In our study, the identification rate in Stage III B \& C (tumors with skin involvement) was nearly $30 \%$.

In 2000, two studies were published concerning the issue of SNLB post neoadjuvant chemotherapy; Nason et al. published their study on 82 patients who underwent SLNB followed by level I-II ALND. SLNs were successfully identified in $80 \%$ of patients with a false negative rate of $33 \%$ (3 patients). They had a conclusion that NAC was associated with high false negative rate [19].

Breslin et al. reported their study conducted on 51 patients with stage II or III breast cancer who received doxorubicin-based neoadjuvant chemotherapy. The study was conducted from 1994 to 1999 with detection rate improving from $64.7 \%$ to $94.1 \%$ and a false-negative rate of $6 \%$ [20].

Stearns et al., in 2002, reported their study conducted on 34 patients with breast cancer after neoadjuvant chemotherapy with detection rate of 85 and a false-negative rate of $14 \%$. Among the subgroup without inflammatory breast cancer, the detection and false-negative rates were $89 \%$ and $6 \%$, respectively. They concluded that SLNB after neoadjuvant chemotherapy may accurately predict axillary staging except in inflammatory breast cancer [21].

In our study, the detection rate equals $82.2 \%$ (37/45). The only significant non-technical factor which could be identified as a possible cause for non-identification of sentinel LNs was persistence of skin manifestations (especially peau de orange) after neoadjuvant chemotherapy in patients who were first presented by T4 lesions before chemotherapy. When T1-3 \& T4 tumors (after NAC) identification rate was calculated independently, the result differed radically; in T1-3 tumors (after NAC); SLN was identified in 34 out of 35 patients giving percentage of $97.1 \%$ and in T4 tumors (after NAC); SLN was identified only in 3 out of 10 patients giving percentage of $30 \%$.

The most probable explanation for that result is that one of the pathological characters of T4 breast cancer is invasion of dermal lymphatic by tumor emboli, which can lead to lymphatic obstruction causing the clinical picture of peau d'orange, rapid breast enlargement and diffuse erythema [22]

This copes with the recently published systematic review and meta-analysis of the accuracy \& feasibility of SLNB after NAC in locally advanced breast cancer enrolling 7451 patients were included. Identification rate was $89.6 \%$, which is lower than the $95 \%$ identification rate observed in recent series of early breast cancer. These findings highlight the limits of SNB performance in this population [23].

The factors affecting the rate of false negative results during are multiple including SLNB technique, variability in lymphatic drainage pathway, level of surgeon experience and patient selection [24].

Among many factors affecting false negative result (fNR), increased surgical experience as well as choosing suitable technique of SLNB may bring down the high fNR to an acceptable minimum [25].

According to the ACOSOG Z1071 trial, factors affecting the accuracy of SLNB 
after neoadjuvant chemotherapy could be identified. Tumor factors (clinical T or $\mathrm{N}$ stage), patient factors (age, BMI), length of chemotherapy treatment, nodal response to chemotherapy and site of injection did not significantly affect the SLN identification rate. The SLN identification rate was higher when mapping was performed using a dual-agent mapping technique vs a single-agent mapping technique. It was also found that the false negative rate was lower when 3 or more SLNs are examined vs only 2 SLNs [26] [27].

Reports from the Dutch breast cancer trialists' group (BOOG), on their study on 657 patients, showed that in patients who converted after neoadjuvant chemotherapy from $\mathrm{cN}+$ to $\mathrm{cN} 0$, the false negative rate was $20 \%$ [28].

In our study, false negative rate equals $52 \%$ which is too high in comparison to other published studies. False negative rate for T4 group equals $69 \%$ while in T1-3 group equals $28 \%$

This may be attributed to: Relatively small sample size, Lymphatic obstruction in the T4 group and, may be, the presence of learning curve; the two false negative cases in T1-3 group were in the first half of the study, in the second half, the fNR for T1-3 group was $0 \%$.

The main Limitations of our study were the relative small sample size, Lymphatic obstruction in the T4 group which represents 22 patients out of the 45 included in the study (48.8\%). This high ratio can be explained by absence of widely applied screening programs and delayed seeking of medical advice by many patients which lead to facing an advanced tumor at the time of diagnosis, and lastly, Presence of learning curve; the two false negative cases in T1-3 group were in the first half of the study, in the second half, the fNR for T1-3 group was $0 \%$.

\section{Conclusion}

SLNB should be adopted to be the standard method for axillary staging in breast cancer patients with T1-3 tumors after receiving neoadjuvant chemotherapy while SLNB is patients with T4 breast cancer after neoadjuvant chemotherapy is associated with low detection rate $\&$ high false negative rate making it doubtful technique for axillary staging.

\section{References}

[1] Gherghe, M., Bordea, C. and Blidaru, A. (2014) Sentinel Lymph Node Biopsy (SLNB) vs. Axillary Lymph Node Dissection (ALND) in the Current Surgical Treatment of Early Stage Breast Cancer. Journal of Medicine and Life, 8, 176-180.

[2] Khavanin, N., Gart, M.S., Berry, T., Thornton, B., Saha, S. and Kim, J.Y. (2014) Sentinel Lymph Node Biopsy versus Axillary Lymphadenectomy in Patients Treated with Lumpectomy: An Analysis of Short-Term Outcomes. Annals of Surgical Oncology, 21, 74-80. https://doi.org/10.1245/s10434-013-3248-3

[3] Tsujimoto, M. (2015) Recent Advances in Sentinel Node Biopsy in Breast Surgery. Breast Cancer, 22, 211. https://doi.org/10.1007/s12282-015-0601-3

[4] Holmes, D., Colfry, A., Czerniecki, B., Dickson-Witmer, D., Espinel, C. F., Feldman, E. and Kuerer, H. (2015) Performance and Practice Guideline for the Use of 
Neoadjuvant Systemic Therapy in the Management of Breast Cancer. Annals of Surgical Oncology, 22, 3184-3190. https://doi.org/10.1245/s10434-015-4753-3

[5] Mamounas, E.P. (2015) Impact of Neoadjuvant Chemotherapy on Locoregional Surgical Treatment of Breast Cancer. Annals of Surgical Oncology, 22, 1425-1433. https://doi.org/10.1245/s10434-015-4406-6

[6] Benson, J.R. and Jatoi, I. (2014) Sentinel Lymph Node Biopsy and Neoadjuvant Chemotherapy in Breast Cancer Patients. Future Oncology, 10, 577-586.

https://doi.org/10.2217/fon.13.231

[7] Yagata, H., Yamauchi, H., Tsugawa, K., Hayashi, N., Yoshida, A., Kajiura, Y. and Nakamura, S. (2013) Sentinel Node Biopsy after Neoadjuvant Chemotherapy in Cytologically Proven Node-Positive Breast Cancer. Clinical Breast Cancer, 13, 471-477. https://doi.org/10.1016/j.clbc.2013.08.014

[8] Srivastava, A. and Misra, M. (2012) In Search of a Cost-Effective Sentinel Node Biopsy Technique: A Randomized Trial Comparing the Use of Methylene Blue and Patent Blue Violet to Identify Sentinel Node in Breast Cancer. Cancer Research, 72, 2705-2705. https://doi.org/10.1158/1538-7445.AM2012-2705

[9] Giuliano, A.E., Kirgan, D.M., Guenther, J.M. and Morton, D.L. (1994) Lymphatic Mapping and Sentinel Lymphadenectomy for Breast Cancer. Annals of Surgery, 220, 391. https://doi.org/10.1097/00000658-199409000-00015

[10] Krag, D., Weaver, D., Alex, J., Fairbank, J., et al. (1993) Surgical Resection and Radiolocalization of the Sentinel Lymph Node in Breast Cancer Using a Gamma Probe. Surgical Oncology, 2, 335-340. https://doi.org/10.1016/0960-7404(93)90064-6

[11] Motomura, K. (2015) Sentinel Node Biopsy for Breast Cancer: Past, Present, and Future. Breast Cancer, 22, 212-220. https://doi.org/10.1007/s12282-012-0421-7

[12] Varghese, P., Mostafa, A., Abdel-Rahman, A., Akberali, S., Gattuso, J., Canizales, A. and Carpenter, R. (2007) Methylene Blue Dye versus Combined Dye-Radioactive Tracer Technique for Sentinel Lymph Node Localisation in Early Breast Cancer. European Journal of Surgical Oncology (EJSO), 33, 147-152. https://doi.org/10.1016/j.ejso.2006.09.026

[13] Schwartz, G.F. and Meltzer, A.J. (2003) Accuracy of Axillary Sentinel Lymph Node Biopsy Following Neoadjuvant (Induction) Chemotherapy for Carcinoma of the Breast. The Breast Journal, 9, 374-379. https://doi.org/10.1046/j.1524-4741.2003.09502.x

[14] Xing, Y., Foy, M., Cox, D., Kuerer, H., Hunt, K. and Cormier, J. (2006) Meta-Analysis of Sentinel Lymph Node Biopsy after Preoperative Chemotherapy in Patients with Breast Cancer. British Journal of Surgery, 93, 539-546. https://doi.org/10.1002/bjs.5209

[15] Kelly, A.M., Dwamena, B., Cronin, P. and Carlos, R.C. (2009) Breast Cancer: Sentinel Node Identification and Classification after Neoadjuvant Chemotherapy-Systematic Review and Meta Analysis. Academic radiology, 16, 551-563. https://doi.org/10.1016/j.acra.2009.01.026

[16] van Deurzen, C.H., Vriens, B.E., Tjan-Heijnen, V.C., van der Wall, E., Albregts, M., van Hilligersberg, R. and Diest, P.J. (2009) Accuracy of Sentinel Node Biopsy after Neoadjuvant Chemotherapy in Breast Cancer Patients: A Systematic Review. European Journal of Cancer, 45, 3124-3130. https://doi.org/10.1016/j.ejca.2009.08.001

[17] Kuehn, T., Bauerfeind, I., Fehm, T., Fleige, B., Helms, G., Lebeau, A. and Schrenk, P. (2012) Abstract S2-2: Sentinel Lymph Node Biopsy Before or after Neoadjuvant Chemotherapy-Final Results from the Prospective German, Multiinstitutional 
SENTINA-Trial. Cancer Research, 72, S2-2-S2-2.

https://doi.org/10.1158/0008-5472.SABCS12-S2-2

[18] Tsuyuki, S., Yamaguchi, A., Kawata, Y. and Kawaguchi, K. (2015) Assessing the Effects of Neoadjuvant Chemotherapy on Lymphatic Pathways to Sentinel Lymph Nodes in Cases of Breast Cancer: Usefulness of the Indocyanine Green-Fluorescence Method. The Breast, 24, 298-301. https://doi.org/10.1016/j.breast.2015.02.034

[19] Nason, K.S., Anderson, B.O., Byrd, D.R., Dunnwald, L.K., Eary, J.F., Mankoff, D.A. and Yeung, R.S. (2000) Increased False Negative Sentinel Node Biopsy Rates after Preoperative Chemotherapy for Invasive Breast Carcinoma. Cancer, 89, 2187-2194.

[20] Breslin, T.M., Cohen, L., Sahin, A., Fleming, J.B., Kuerer, H.M., Newman, L.A. and Feig, B.W. (2000) Sentinel Lymph Node Biopsy Is Accurate after Neoadjuvant Chemotherapy for Breast Cancer. Journal of Clinical Oncology, 18, 3480-3486. https://doi.org/10.1200/JCO.2000.18.20.3480

[21] Stearns, V., Ewing, C.A., Slack, R., Penannen, M.F., Hayes, D.F. and Tsangaris, T.N. (2002) Sentinel Lymphadenectomy after Neoadjuvant Chemotherapy for Breast Cancer May Reliably Represent the Axilla except for Inflammatory Breast Cancer. Annals of surgical oncology, 9, 235-242. https://doi.org/10.1007/BF02573060

[22] Kwon, S., Agollah, G., Wu, G. and Sevick-Muraca, E.M. (2015) Abstract P5-01-04: In vivo Lymphatic Imaging of a Human Inflammatory Breast Cancer Model. Cancer Research, 75, P5-01-04. https://doi.org/10.1158/1538-7445.SABCS14-P5-01-04

[23] Mocellin, S., Goldin, E., Marchet, A. and Nitti, D. (2015) Sentinel Node Biopsy Performance after Neoadjuvant Chemotherapy in Locally Advanced Breast Cancer: A Systematic Review and Meta-Analysis. International Journal of Cancer, 138, 472-480. https://doi.org/10.1002/ijc.29644

[24] Wang, Y., Dong, H., Wu, H., Zhang, L., Yuan, K., Chen, H. and Fu, R. (2015) Improved False Negative Rate of Axillary Status Using Sentinel Lymph Node Biopsy and Ultrasound-Suspicious Lymph Node Sampling in Patients with Early Breast Cancer. BMC Cancer, 15, 382. https://doi.org/10.1186/s12885-015-1331-9

[25] Sekhar, G.C., Vamseedhar, K. and Babu, M.H. (2015) A Study of Sentinel Lymph Node Biopsy in Early Breast Cancer Using Methylene Blue in a Tertiary Care Hospital in South India. IOSR Journal of Dental and Medical Sciences (IOSR-JDMS), Dr. NTR University of Health Sciences, Vijayawada.

[26] Boughey, J.C., Suman, V.J., Mittendorf, E.A., Ahrendt, G.M., Wilke, L.G., Taback, B. and Bowling, M. (2015) Factors Affecting Sentinel Lymph Node Identification Rate after Neoadjuvant Chemotherapy for Breast Cancer Patients Enrolled in ACOSOG Z1071 (Alliance). Annals of Surgery, 261, 547-552.

https://doi.org/10.1097/SLA.0000000000000551

[27] Boughey, J.C., Suman, V.J., Mittendorf, E.A., Ahrendt, G.M., Wilke, L.G., Taback, B. and Flippo-Morton, T.S. (2013) Sentinel Lymph Node Surgery after Neoadjuvant Chemotherapy in Patients with Node-Positive Breast Cancer: The ACOSOG Z1071 (Alliance) Clinical Trial. JAMA, 310, 1455-1461.

https://doi.org/10.1001/jama.2013.278932

[28] Tjan-Heijnen, V.C., Vriens, B.E., Aarts, M.J., Kroep, J.R., van de Velde, C.J., Liefers, G.-J. and de Boer, M. (2015) Abstract P2-01-03: Sentinel Node Procedure before or after Neoadjuvant Chemotherapy in Clinically Node Negative or Positive Patients; Results from 3 Phase III Studies of the Dutch Breast Cancer Trialists' Group (BOOG). Cancer Research, 75, P2-01-03.

https://doi.org/10.1158/1538-7445.SABCS14-P2-01-03 